\title{
Targeting Mitochondria for Cancer Treatment - Two Types of Mitochondrial Dysfunction
}

\author{
Jiří Pokorný1, Jan Pokorný ${ }^{2}$, Jitka Kobilková ${ }^{3}$, Anna Jandová1, \\ Jan Vrba ${ }^{4}$, Jan Vrba Jr. ${ }^{5}$ \\ ${ }^{1}$ Institute of Photonics and Electronics, Academy of Sciences of the Czech Republic, \\ Prague, Czech Republic; \\ ${ }^{2}$ Institute of Physics, Academy of Sciences of the Czech Republic, Prague, \\ Czech Republic; \\ ${ }^{3}$ Department of Obstetrics and Gynaecology, First Faculty of Medicine, \\ Charles University in Prague and General University Hospital in Prague, Prague, \\ Czech Republic; \\ ${ }^{4}$ Faculty of Electrical Engineering, Czech Technical University in Prague, Prague, \\ Czech Republic; \\ ${ }^{5}$ Faculty of Biomedical Engineering, Czech Technical University in Prague, Kladno, \\ Czech Republic
}

Received July 1, 2014; Accepted November 18, 2014.

Key words: Warburg effect - Reverse Warburg effect - Mitochondrial membrane potential - Ordered water around mitochondria - Uptake and retention of fluorescent dyes - Physics of malignancy

\begin{abstract}
Two basic types of cancers were identified - those with the mitochondrial dysfunction in cancer cells (the Warburg effect) or in fibroblasts supplying energy rich metabolites to a cancer cell with functional mitochondria (the reverse Warburg effect). Inner membrane potential of the functional and dysfunctional mitochondria measured by fluorescent dyes (e.g. by Rhodamine 123) displays low and high values (apparent potential), respectively, which is in contrast to the level of oxidative metabolism. Mitochondrial dysfunction (full function) results in reduced (high) oxidative metabolism, low (high) real membrane potential, a simple layer (two layers) of transported protons around mitochondria, and high
\end{abstract}

This study was supported by COST Action TD1301, MiMed and by the Technological Agency of the Czech Republic, project TA02010854: "Research of Wide Spectrum Light in Medicine".

Mailing Address: Ing. Jiří Pokorný, DSc., Institute of Photonics and Electronics, Academy of Sciences of the Czech Republic, Chaberská 57, 18251 Prague 8, Czech Republic; e-mail: pokorny@ufe.cz 
(low) damping of microtubule electric polar vibrations. Crucial modifications are caused by ordered water layer (exclusion zone). For the high oxidative metabolism one proton layer is at the mitochondrial membrane and the other at the outer rim of the ordered water layer. High and low damping of electric polar vibrations results in decreased and increased electromagnetic activity in cancer cells with the normal and the reverse Warburg effect, respectively. Due to nonlinear properties the electromagnetic frequency spectra of cancer cells and transformed fibroblasts are shifted in directions corresponding to their power deviations resulting in disturbances of interactions and escape from tissue control. The cancer cells and fibroblasts of the reverse Warburg effect tumors display frequency shifts in mutually opposite directions resulting in early generalization. High oxidative metabolism conditions high aggressiveness. Mitochondrial dysfunction, a gate to malignancy along the cancer transformation pathway, forms a narrow neck which could be convenient for cancer treatment.

\section{Introduction}

Energy supply, its transformation, and utilization in biological systems are a hallmark of a living organism. From the physical point of view biological systems are in a coherent state far from the thermodynamic equilibrium which is an essential condition for biological activity and life. Energy is utilized in various processes, for instance, in transport, interactions, organization, and information transfer. Any elementary motion depends on the energy supply. O. Warburg intuitively assessed the cancer process as a disturbance of the energy transformation and utilization. He experimentally disclosed that cells in the cancer tissue can obtain approximately the same amount of energy from fermentation as from respiration whereas the healthy cells obtain much more energy from oxidation than from fermentation (Warburg et al., 1924;Warburg, 1956). Biological research orientated predominantly to the biochemical and genetic processes considered the Warburg effect to be a side corollary of the cancer process and not its central issue. On the other hand the mechanisms of the energy supply and the role of the biological electromagnetic field (EMG) cannot be understood without deep understanding of the structure of biomolecules, biochemical reactions, and genetic processes. (In biological cells the most significant component of EMG is very likely the microtubule near field which is of electrodynamic nature.) Mitochondria are the boundary elements between the chemical-genetic and the physical processes in cells (Pokorný, 2012; Pokorný et al., 2013b). The energy released from the flux of electrons down the electron transport chain and associated with the production of reactive oxygen species (ROS) is used for transfer of protons from the mitochondrial matrix space across the inner membrane and creation of the electrochemical potential. The protons diffuse through the outer membrane into cytosol. The electric field between the positively charged protons and the negative charge of ROS in the matrix space produces water ordering around mitochondria 
(Pokorný, 2012). A layer of a strong static electric field was measured up to a distance of about $2 \mu \mathrm{m}$ from the surface of mitochondria (Tyner et al., 2007). In the interphase mitochondria are aligned along microtubules and form essential conditions for generation of the EMG by microtubules. Mitochondria supply the energy, the ordered water provides low damping, and the static electric field shifts the microtubule oscillations into a highly non-linear region (Pokorný et al., 2011; Pokorný, 2012). The generated EMG seems to have a fundamental role in biological activity.

$\mathrm{H}$. Fröhlich formulated a hypothesis of coherent electrical polar vibrations in biological systems with energy condensation in one or a few modes of motion correlated over macroscopic region (Fröhlich, 1968a, b, 1969, 1973, 1980).

Disturbances of interactions of a cell in the tissue due to its frequency changes of polar vibrations were assumed to be an initial condition for the origin of cancer malignant activity (Fröhlich, 1978). The generated electrical oscillating field was measured by attraction of small dielectric particles with a high permittivity and linear dimensions about $1 \mu \mathrm{m}$ by yeast cells. This dielectrophoretic attraction disclosed the highest power in the M phase (Pohl et al., 1981). The $M$ phase increased activity of yeast cells in the frequency region $8-9 \mathrm{MHz}$ was confirmed by Pokorný et al. (2001). Sahu et al. (2013) measured resonant frequencies of isolated microtubules in the frequency range $10-30 \mathrm{MHz}, 100-200 \mathrm{MHz}$, at about $20 \mathrm{THz}$ (the wavenumber about $700 \mathrm{~cm}^{-1}$ ), and the UV absorption spectrum at about $270 \mathrm{~nm}$. Advanced biological research assumes that the tissue organization field is based on biomechanical forces generated in and by tissues (Soto and Sonnenschein, 2011). Morphogenesis is also discussed on the basis of a model of mechanical forces generated in the system (Beloussov, 2012). The physical nature and origin of the suggested forces are not specified. Other authors assume that orchestrated activities are mediated by bioelectric signals based on voltage gradients of the transmembrane potential (Levin, 2012, 2014; Chernet and Levin, 2014).

Examination of the glycolytic phenotype cancer cells, i.e. cancer cells with the inhibited pyruvate pathway in mitochondria, the high uptake and retention of fluorescent dyes by mitochondria (hyperpolarization), and restoration of their healthy state was performed and published by Bonnet et al. (2007). The lack of hyperpolarization of the mitochondrial membranes in some cancer cells (Chen, 1988) suggests another type of the mitochondrial dysfunction and apoptosis blocking (Pavlides et al., 2009). The cancer cells with the high activity of mitochondria and the mitochondrial dysfunction in fibroblasts in the stromal microenvironment may produce this type of the energy production defects. Analysis of the cancers with the reverse Warburg effect presented in this article forms an additional part to the description of the glycolytic phenotype cancers (Pokorný et al., 2012a, b, 2013a).

Energy supply is a crucial condition of life and mitochondrial oxidative metabolism sets up its higher forms. Disturbances of the coherent state far from 
the thermodynamic equilibrium caused by the mitochondrial dysfunction are considered to be an essential point of cancer transformation (Pokorný et al., 2013b, 2014). Analysis of the published data on the potential of the mitochondrial inner membrane, the normal and the reverse Warburg effect, and dependence of the ordered water layer on $\mathrm{pH}$ concentration proves that the measured potential may be modified by the ordered water layer, which is a novel contribution explained in this article. The result enables a rational assignment of the apparent potentials to both types of the Warburg effect. The high and the low value of the apparent membrane potential correspond to the low and the high mitochondrial oxidative activity of the cancer cell, respectively. Consequently, the cancer cells with the reverse Warburg effect have a high power of the EMG and high biological activity, which explains their high aggressiveness.

\section{Mitochondrial inner membrane potential}

Warburg disclosed perturbations of the oxidative metabolic energy production connected with cancer transformation - the dysfunction of mitochondria. The functional mitochondria form a negative potential across the inner membrane dependent on the distribution of the negative and positive charges connected with their metabolic activity (Klingenberg and Rottenberg, 1977). The uptake and retention of positively charged fluorescent dyes by mitochondria were used for measurement of the mitochondrial membrane potential. One highly specific dye for this purpose is Rhodamine 123 (Rh123) with a delocalized positive charge (Chen, 1988; O'Connor et al., 1988). Dependence of the uptake and retention of Rh123 on the negative potential was measured on isolated mitochondria (Modica-Napolitano and Aprille, 1987). The level of the absorbance and fluorescence values is a function of the membrane potential (Chen, 1988). (With respect to the further analysis the potential measured by the uptake and retention of fluorescence dyes is termed the apparent potential.)

A correlation between the apparent potential and the real negative potential is essential for assessment of its variations under different conditions. The membrane potential depends on various factors including, for instance, cell growth (Goldstein and Korczack, 1981; Chen, 1988), distribution of ions (Klingenberg and Rottenberg, 1977), and ionophores (Reed, 1979). During continuous respiration, some compounds such as nigericin hyperpolarize mitochondria by electrically neutral exchange of protons for potassium ions leading to decrease of the $\mathrm{pH}$ gradient and increase of the membrane potential in compensation for the change of the electrochemical potential (Reed, 1979; Johnson et al., 1982). The measurement method using a fluorescent dye was applied to the assessment of differences between the healthy and cancer cells. Mitochondria in normal healthy cells display a low apparent potential (Nadakavukaren et al., 1985; Chen, 1988). In contrast a great majority of adenocarcinoma, transitional cell carcinoma, squamous cell carcinoma, and melanoma have a high apparent potential (Lampidis et al., 1983; Chen, 1988). 
A profound relationship between differences in the mitochondrial apparent membrane potential and the tumorigenic properties was found. The apparent potential makes possible determination of the probability of the colonic tumor expansion and progression (Heerdt et al., 2003, 2006).

The negative-inside inner membrane potential generated upon energization of the mitochondria depends on the proton transfer from the matrix space. The equilibrium distribution of ${ }^{86} \mathrm{Rb}$ atoms on both sides of the membrane and the Nernst equation (Modica-Napolitano and Aprille, 1987) were used to calibrate the fluorescence data supplied from uptake and retention of Rh123. The absolute values of the apparent potential of the cancer cells CX-1 and MIP101 were about $60 \mathrm{mV}$ higher than the values of the apparent potential of the healthy cells CV-1 (Modica-Napolitano and Aprille, 1987). The inhibitory effect of the Rh123 fluorescent dye on the mitochondrial bioenergetics function was demonstrated with the conclusion that $\mathrm{F}_{0} \mathrm{~F}_{1}$-ATPase was the primary site of toxicity (ModicaNapolitano et al., 1984; Modica-Napolitano and Aprille, 1987). The increased apparent potential (i.e. hyperpolarization of the inner membrane compared to healthy cells) examined by the tetramethyl rhodamine methyl ester (TMRM) was observed on several types of cancer cells with the inhibited pyruvate transfer into mitochondria (Bonnet et al., 2007). Restoration of the pyruvate transfer by application of dichloroacetate (DCA) results in increased oxidative mitochondrial activity, reversal of hyperpolarization, and return of the potential to the level of healthy cells. Similar results were measured by a protonophore (carbonylcyanidep-trifluoromethoxyphenylhydrazone, FCCP). This experiment disclosed that the high apparent potential is connected with a low oxidative energy production and vice versa. However, the real mitochondrial membrane potential should depend on the proton transfer, i.e. the high membrane potential has to correspond to a high oxidative activity. A possible dependence of the apparent mitochondrial potential on water ordering is explained in Discussion. It should be mentioned that cancer cells may be selectively damaged by changes of mitochondrial ultrastructure (Modica-Napolitano et al., 1996).

However, not all cancer transformations correspond to the model of hyperpolarized mitochondrial membrane and may differ in this feature. For comparison, two opposite cases are presented. For instance, v-fos oncogene transformed fibroblasts have been shown to have higher apparent potential in comparison with their untransformed counterparts (Zarbl et al., 1987). In contrast, $\mathrm{v}$-fes oncogene transformed mink fibroblasts display change from a state of high apparent potential and low $\mathrm{pH}$ gradient to a state of a very low apparent potential and a very high $\mathrm{pH}$ gradient (Johnson et al., 1982; Chen, 1988). Mitochondria in two human colon carcinoma cell lines had the absolute value of the apparent potential lower than the potential of their healthy counterparts and nigericin failed to hyperpolarize the mitochondria (Modica-Napolitano et al., 1989). A short overview of cancers with low and high apparent potential of mitochondria is in Table 1. 


\section{Table 1 - Mitochondrial inner membrane apparent potential of the healthy and the cancer cells}

\begin{tabular}{|c|c|c|}
\hline Low apparent potential & $\begin{array}{l}\text { High apparent potential } \\
\text { (hyperpolarized mitochondria) }\end{array}$ & $\begin{array}{l}\text { Low or very low } \\
\text { apparent potential }\end{array}$ \\
\hline Normal epithelial cells & $\begin{array}{l}\text { A great majority of: } \\
\text { - adenocarcinoma } \\
\text { - transitional cell carcinoma } \\
\text { - squamous cell carcinoma } \\
\text { - melanoma }\end{array}$ & $\begin{array}{l}\text { - human oat cells } \\
\text { - large cell carcinoma of lung } \\
\text { - poorly differentiated carcinoma } \\
\text { of the colon } \\
\text { - leukemias } \\
\text { - lymphomas } \\
\text { - neuroblastomas } \\
\text { - osteosarcomas }\end{array}$ \\
\hline $\begin{array}{l}\text { Low } \mathrm{Rh} 123 \text { uptake } \\
\text { and retention }\end{array}$ & $\begin{array}{l}\text { High Rh123 uptake } \\
\text { and retention }\end{array}$ & $\begin{array}{l}\text { Low or very low Rh123 } \\
\text { uptake and retention }\end{array}$ \\
\hline
\end{tabular}

The data are taken from: Klingenberg and Rottenberg (1977), Modica-Napolitano and Aprille (1987), Chen (1988), O'Connor et al. (1988). Rh123 - Rhodamine 123

The experimental data of the apparent potential suggest that mitochondria in cancer cells may be in two different states corresponding to the apparent potential: one state connected with inhibited and the other state with normal or highly active mitochondrial function. Analysis of cancer cells with a high value of the apparent potential was performed by Bonnet et al. (2007). Pyruvate transfer into the matrix space is inhibited by phosphorylation of the pyruvate dehydrogenase (PDH) enzyme family by the pyruvate dehydrogenase kinases (PDKs). As a consequence, the diminished flux of electrons along the electron transfer chain may result not only in the decreased efflux of protons from the matrix space but also in the reduced production of reactive oxygen species (ROS). The release of ROS regulates the opening of the plasma membrane ion channels. Downregulation of the $\mathrm{K}^{+}$channel $\mathrm{Kv} 1.5$ results in the increased concentration of $\mathrm{K}^{+}$ions inside the cell by the decrease of their efflux down their intracellular/extracellular gradient. For instance, production of the mitochondria-derived relatively stable ROS- $\mathrm{H}_{2} \mathrm{O}_{2}$ is diminished with the consequent inactivation of $\mathrm{Kv} 1.5$. The proton space charge field together with the water ordering is disturbed. Opening the pyruvate transfer restores normal conditions for the mitochondrial function.

\section{Cancer cells with highly active mitochondrial function}

Cancer cells with the inhibited pyruvate transfer into the mitochondrial matrix space represent only one possible type of the mitochondrial dysfunction. Another type of energy production defects may exist in cancers. Cancer cell effects on stroma were observed and suggested to constitute a separate mechanism of response of myofibroblasts to the invasive neoplasia (Seemayer et al., 1979). It was also assumed that malignant tumor cells and adjacent "normal" cells have to undergo a mysterious cancerization process as was mentioned by Martinez- 
Outschoorn et al. (2010a). Pavlides et al. (2009, 2010a, b) disclosed that the cancer associated fibroblasts secrete the energy rich metabolites produced by the aerobic glycolysis. The epithelial cancer cells take them up and use them in a highly effective mitochondrial ATP production via oxidative phosphorylation. This mechanism is called the reverse Warburg effect. The reverse Warburg effect was observed in breast cancers, advanced prostatic cancers, and may exist in many different types of epithelial cancers dependent on tumor microenvironment (Pavlides et al., 2009, 2010a; Bonuccelli et al., 2010a, b; Martinez-Outschoorn et al., 2010a). An overview of the healthy and the cancer cells with different mitochondrial potential is in Table 1. One of the essential conditions for creation of the reverse Warburg effect is a loss of the stromal caveolin-1 (Cav-1) expression (Pavlides et al., 2009, 2010a; Bonuccelli et al., 2010b; Chiavarina et al., 2010; Martinez-Outschoorn et al., 2010b; Ko et al., 2011), which is connected with induction of the oxidative stress, autophagy/mitophagy (Pavlides et al., 2010a; Martinez-Outschoorn et al., 2010b, 2011), and the mitochondrial dysfunction in the associated fibroblasts (Pavlides et al., 2009). [The activated myo-fibroblasts perform a normal wound healing function which is mediated via differentiation of the normal fibroblasts (Pavlides et al., 2009). Their lactate production and secretion suggest that the mechanism of the reverse Warburg effect may be a general feature of their activity.] The normal epithelial cells undergo an epithelial-mesenchymal transition (Bonuccelli et al., $2010 \mathrm{~b})$. The tumor growth and metastasis are fuelled by the supply of energy rich metabolites, such as lactate, pyruvate, glutamine, keton BHB (beta-hydroxybutyrate) from the associated fibroblasts (Pavlides et al., 2009, 2010a; Bonuccelli et al., 2010a; Migneco et al., 2010; Ko et al., 2011). Supply of the energy rich metabolites (Martinez-Outschoorn et al., 2011), loss of Cav-1 in the stromal fibroblasts (Martinez-Outschoorn et al., 2010a) and their excess production of ROS drive an anti-oxidant defence in the cancer cells and protection against apoptosis (Lisanti et al., 2010; Martinez-Outschoorn et al., 2010b).

The mechanism of fibroblast degradation for production and secretion of the energy rich metabolites depends on the ROS production by the cancer cell. Cav-1 is an inhibitor of the nitric oxide (NO) production in fibroblasts (Lisanti et al., 2010). A loss of Cav-1 causes the increased NO production, which leads to the mitochondrial dysfunction, the increased ROS production, and the enlarged oxidative stress (Lisanti et al., 2010; Martinez-Outschoorn et al., 2010a). The glycolytic enzyme isoform of pyruvate kinase (PKM2) and the lactate dehydrogenase B (LDH B) mediate the reverse Warburg effect (Bonuccelli et al., 2010b). The oxidative stress in the neighbouring stromal cells may be also induced by hydrogen peroxide $\left(\mathrm{H}_{2} \mathrm{O}_{2}\right)$ secreted by the cancer cells (Sotgia et al., 2012; Witkiewicz et al., 2012). The tumor-stroma co-evolution is described, for instance, by Lisanti et al. (2010).

The tumors with the fully functional mitochondria and supply of the energy rich metabolites from the fibroblasts have increased risk of the recurrence, the 
lymph node metastasis, the treatment drug resistance, and a poor clinical outcome. Inhibitors of glycolysis prevent the tumor promoting effects. Combination of 2-DG (2-deoxy-D-glucose) with DCA (dichloroacetate) - two well established glycolysis inhibitors - may strongly reduce the tumor growth (Bonuccelli et al., 2010b).

\section{Discussion}

The pathological breakdown of the coherent energy states far from the thermodynamic equilibrium is considered to be a general process of cancer (Pokorný et al., 2013b, 2014). The mitochondrial dysfunction in cancer cells or the cancer cell associated stromal fibroblasts plays an essential role in cancer transformation and development. The electromagnetic field generated by microtubules depends on the mitochondrial function (Pokorný, 2012; Pokorný et al., 2012a, b). In healthy cells the mitochondria supply energy, provide low damping of oscillations due to the ordered water around them, and produce a strong static electric field shifting the microtubule oscillations into the nonlinear region. The oscillation frequencies depend on the nonlinear characteristic of the system. If the force constant in the potential valley increases with the decreasing oscillation power the frequency increases (with the increasing power the frequency decreases). The absorption resonant frequencies of some tumors were measured around $465 \mathrm{MHz}$ (Vedruccio and Meessen, 2004), which corresponds to the shifted spectral lines of the healthy cells in the frequency band below $200 \mathrm{MHz}$.

In the case of the Warburg effect the power of microtubule oscillations and of the generated electromagnetic field in cancer cells may be downregulated and its parameters changed. Coherence of the electromagnetic field may be

\begin{tabular}{|c|c|c|c|}
\hline & & Cancer transformation pathway & \multirow[b]{2}{*}{ Cancer } \\
\hline Initial & state & Precancerous state & \\
\hline $\begin{array}{l}\text { Decreased } \\
\text { genome } \\
\text { stability }\end{array}$ & $\begin{array}{l}\text { Oncogene } \\
\text { mutation } \\
\text { Induction }\end{array}$ & $\begin{array}{l}\text { Warburg effect } \\
\text { MD in cancer cell or } \\
\text { reverse Warburg effect }\end{array}$ & $\begin{array}{l}\text { Rebuilt and shifted } \\
\text { frequency spectrum }\end{array}$ \\
\hline $\begin{array}{l}\text { (Disturbance } \\
\text { of coherent } \\
\text { states?) }\end{array}$ & $\begin{array}{l}\text { of PDK 1-4 } \\
\text { Inhibition } \\
\text { of PDP 1-2 }\end{array}$ & $\begin{array}{l}\text { MD in associated } \\
\text { fibroblasts }\end{array}$ & $\begin{array}{l}\text { Escape from tissue } \\
\text { interaction and contro }\end{array}$ \\
\hline
\end{tabular}

Figure 1 - A diagram of a cancer transformation pathway with the Warburg effect, the reverse Warburg effect, and a hypothetical link of the changed electromagnetic activity. The rebuilt and shifted frequency spectrum of the cancer cells makes possible their escape from tissue control and regulation. Mitochondrial dysfunction (MD) is formed either in the cancer cells or in the associated fibroblasts. The cancer transformation pathway is divided into three essential parts: the initial state containing disturbances of the biochemical and genetic mechanisms (genome mutations), the precancerous state when the mitochondrial dysfunction is created, and the final cancer part with a hypothetical explanation of malignant properties. It is assumed that a massive genome mutation depends on the low level of coherent energy states. 


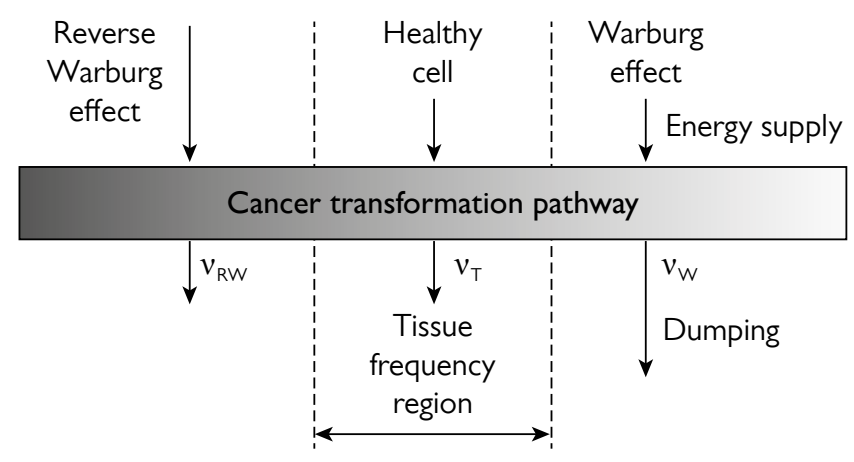

Figure 2 - A schematic picture of hypothetical frequency spectrum shifts of the electromagnetic field generated by microtubules in cancer cells. The central part corresponds to a normal healthy cell and the side parts to cancer cells. The frequency spectrum shifts depend on the power of microtubule oscillations and the generated electromagnetic field. The power of the electromagnetic field generated by the cancer cells with the normal and the reverse Warburg effect is lower than and equal to or higher than the power of the healthy cells, respectively. $v_{W}$ and $v_{R W}$ denote frequencies of the spectra of cancer cells with the normal and the reverse Warburg effect, respectively. $v_{T}$ stands for the frequencies of the spectrum of the healthy tissue cells. Damping depends on the level of water ordering conditioned by mitochondrial function.

disturbed, the frequency spectrum changed and shifted from the tissue frequency region (in correspondence to the decreased power). In the case of the reverse Warburg effect the enhanced mitochondrial activity and supply of the energy rich metabolites to cancer cells provide the increased power of oscillations, the changed frequency spectrum and its shift outside the tissue region too (in correspondence to the increased power). Consequently, in both types of the Warburg effects the cancer cells may escape from the tissue control and regulations and begin their activity independent of the tissue. Figure 1 shows a cancer transformation pathway (with a hypothetical term of the disturbed electromagnetic field) with the mitochondrial dysfunction in the cancer cells or in the associated fibroblasts. In the case of the Warburg effect and the reverse Warburg effect the frequency spectrum of the electromagnetic field is shifted outside the tissue frequency region, very likely to the higher and lower frequencies, respectively. Cancer cells escape from the tissue control and regulation (Figure 2). Due to opposite frequency shifts of EMG in the cancer cells and their associated fibroblasts a tendency towards local invasion and metastasis should be increased in cancers with the reverse Warburg effect at their early stage of development. These cancers are aggressive, with increased risk of recurrence, and poor clinical outcome (Chiavarina et al., 2010).

Infection with the lactate dehydrogenase elevating virus (LDHV) increases the level of the lactate dehydrogenase (LDH, now classified as NAD 1.1.1.27 Oxidoreductase) isoenzymes in plasma. LDH isoenzymes are upregulated in the fibroblastic stromal compartment of the human breast cancer samples that lack 
the stromal Cav-1 expression (Pavlides et al., 2009; Bonuccelli et al., 2010a). LDH-B may be a novel biomarker for the reverse Warburg effect (Bonuccelli et al., 2010b). An extensive investigation of the role of LDH isoenzymes in the cancer process was performed. Activity of the LDH isoenzymes (fractions) was determined by the electrophoretic method using agar gel. The blood serum of cancer patients with the gynaecological tumors (for instance, of vulva, endometrium, ovarium, cervix) and the tumorous tissues were examined. An increased activity level of the LDH 5 isoenzyme in the majority of the cancer patients was observed by Škoda et al. (1967) and Jandová et al. (1969a, b, 1970, 1971). (The LDH enzyme isoforms are marked either by capital letters or numerals.)

The cell-mediated immunity response to the LDHV antigen was examined too. The LDHV antigen was prepared from the serum of inbred mice of the $\mathrm{C} 3 \mathrm{H} \mathrm{H}^{2 \mathrm{k}}$ strain infected with the LDHV.As a response to the LDHV antigen the $T$ lymphocytes adherence to solid surfaces manifests changes which are evaluated in healthy humans and cancer patients with different cancers. A positive response to the LDHV antigen was a general feature of the investigated cases of the cancer process (Jandová et al., 2001). Using the described method T lymphocytes prepared from the blood of the cervical precancerous patients were examined. The results lead to a conclusion that in the cervical cancer the glycolytic and the mitochondrial oxidative energy production are transformed in the precancerous link of cancer transformation, which is the last step before local invasion and metastasis (Jandová et al., 2009).

Electric polarization of the mitochondrial membrane depends on the production of ROS and the proton transport from the matrix space across the membrane, on the distribution of ions in the cell, and on other conditions inside the cell. Effects of cell growth, varying inhibition of the mitochondrial function by the fluorescent dyes, plasma membrane potential, and ionophores on the apparent membrane potential were investigated. The main effect was assigned to inhibition of the Kv1.5 channels in the plasma membrane resulting in the increased concentration of $\mathrm{K}^{+}$ in the cell (Bonnet et al., 2007). However, the effect of the water ordering around mitochondria (Pokorný, 2012) has not been adequately explained.

The apparent potential (negative inside the matrix) of mitochondria in the cancer cells is higher than and equal to (or even smaller than) the apparent potential of their healthy counterparts for the low and high oxidative activity, respectively. Consequently, the apparent potential differs from the real potential which is proportional to mitochondrial oxidative activity - the low and high value of the real membrane potential depends on the low and high metabolic activity, respectively. However, the experimental data from the fluorescent measurement of the potential across the inner membrane were interpreted without taking into account the ordered water layer around mitochondria. The thickness of the ordered water layer forming exclusion zone depends on $\mathrm{pH}$ (Zheng and Pollack, $2003,2006)$, the membrane potential, and the type and concentration of ions in the 
solution. The exclusion zone is not formed for positively charged particles and a negative membrane potential corresponding to $\mathrm{pH}$ equal to and greater than a limit value as follows from analysis of the experimental findings of Zheng and Pollack (2003). The exclusion zone is formed and its thickness increases with decreasing $\mathrm{pH}$ in the region below its limit. Therefore, for a low membrane potential $(\mathrm{pH}$ is greater than the limit value) the transported protons from the matrix form a simple layer around the mitochondrion. For a high membrane potential $(\mathrm{pH}$ is below the limit value) two proton layers may be formed: a) an inner proton layer at the membrane below the ordered water layer, where the electric forces exerted on protons by the matrix negative charge are greater than the forces exerted by diffusion and the ordered water layer, b) a proton layer at the outer rim of the ordered water. In the latter case the number of protons in the layer is smaller than the total number of transported protons but their number is a function of the membrane potential. The fluorescent dye molecules very likely cannot replace protons in the inner layer between the membrane and the ordered water layer. Therefore, the apparent membrane potential depends either on the replacement of the protons in the simple layer or in the layer at the outer rim of the ordered water by fluorescent dye molecules. At the low mitochondrial oxidative activity the number of protons in the simple layer may be greater than their number in the layer at the outer rim in the case of high oxidative activity. Therefore, the number of the replaced protons by the molecules of a fluorescent dye might not correspond to the real membrane potential. The low or the very low apparent potential is measured on mitochondria with the high or very high proton transfer across the membrane, respectively. Cancer cells with the reverse Warburg effect and a hyperactive mitochondrial function have a low or a very low apparent potential.

Mitochondrial dysfunction is a central process along the cancer transformation pathway. The cell mobilizes the tumor suppressor networks to avert malignant transformation using oncogene-induced senescence (OIS). The crucial mediator of the OIS is the mitochondrial gatekeeper - the pyruvate dehydrogenase (PDH) enzyme family. Induced OIS keeps a normal function of PDH and abrogated OIS results in the mitochondrial dysfunction by inhibition of the pyruvate transfer (Kaplon et al., 2013; Olenchock and Vander Heiden, 2013). The mitochondrial dysfunction may develop in the cancer cell or in its associated stromal fibroblasts. In the former case the power of the generated electromagnetic field of cancer cells is lower (with low level of coherence) and in the latter case equal to or higher than power in a corresponding healthy cell.

Cancers of a different origin of the genetic and biochemical disturbances develop to a narrow neck of the transformation pathway - the decrease of oxidative metabolism. The Warburg and the reverse Warburg effect may be breakpoints in the vast majority of cancers for creating malignity which develops after passing this neck to disturbances of the physical processes. The different types of malignancies may be vulnerable in this link and might be destroyed by restoration of the normal 
mitochondrial function in cancer cells and/or their associated fibroblasts. This might be a favourable condition for treatment.

Treatment of the cancer cells with the Warburg effect should restore a normal mitochondrial function. The pyruvate pathway into the mitochondrial matrix is blocked through phosphorylation of the PDH enzymes by PDK's as described by Bonnet et al. (2007). Restoration of the full PDH activity and a normal mitochondrial function may be performed, for instance, by DCA but it is not active for all PDK's (Sun et al., 2010). However, the restored healthy state may not be stable and the cell may return to the cancer version. The pathological production of PDK's caused by the oncogene mutation should be targeted too. Treatment of the cancer cells with the reverse Warburg effect may be rather complicated. Combination of different steps and action on different parts of the system is necessary. First of all the physiological level of the stromal Cav-1 should be very likely excited. The stromal fibroblasts have to restore their mitochondrial function by blocking the cancer cell transformation signals and removing inhibition of pyruvate transfer. Another step consists in cutting off secretion of the energy rich metabolites and their transport to the cancer cell. Special care should be given to restoration of the apoptotic function. But in spite of the intensive research on the reverse Warburg effect cancers, the treatment remains at the initial stage.

\section{Conclusion}

Cancer process involves two general types: cancers with the normal and with the reverse Warburg effect. The normal as well as the reverse Warburg effect transforms the glycolytic and oxidative metabolism in the cells, which may be the last link before the malignant cancer behaviour. In the case of the normal Warburg effect the mitochondria in the cancer cells have a high apparent potential (hyperpolarization) measured by the uptake and retention of the fluorescent dyes. The mitochondria are dysfunctional with oxidative metabolism lowered due to inhibition of the pyruvate transfer. The high value of the apparent potential (hyperpolarization) of mitochondria in the cancer cells with the Warburg effect seems to depend on distribution of protons and other positive charges without a limiting effect of the ordered water layer. The treatment should be targeted to restoring the mitochondrial physiological function and opening the apoptotic pathway.

In the case of the reverse Warburg effect the cancer cells have fully functional mitochondria and supply of the energy rich metabolites (pyruvate, lactate, glutamine, etc.) from the associated fibroblasts with the mitochondrial dysfunction. Cancer cells of this type have high power, large aggressiveness, and high probability of metastatic activity but a low apparent potential across the inner membrane of the mitochondria. The low value is explained by formation of a layer of ordered water separating two proton layers around mitochondria. The treatment should restore the mitochondrial function in the associated fibroblasts and adjust a physiological cooperation in the tissue. 


\section{References}

Beloussov, L.V. (2012) Morphogenesis as a macroscopic self-organizing process. Biosystems 109, $262-279$.

Bonnet, S., Archer, S. L., Allalunis-Turner, J., Haromy, A., Beaulieu, C., Thompson, R., Lee, C. T., Lopaschuk, G. D., Puttagunta, L., Bonnet, S., Harry, G., Hashimoto, K., Porter, C. J., Andrade, M. A., Thebaud, B., Michelakis, E. D. (2007) A mitochondria- $\mathrm{K}^{+}$channel axis is suppressed in cancer and its normalization promotes apoptosis and inhibits cancer growth. Cancer Cell 11, 37-51.

Bonuccelli, G., Tsirigos, A., Whitaker-Menezes, D., Pavlides, S., Pestell, R. G., Chiavarina, B., Frank, P. G., Flomenberg, N., Howell, A., Martinez-Outschoorn, U. E., Sotgia, F., Lisanti, M. P. (2010a) Ketones and lactate "fuel" tumor growth and metastasis. Evidence that epithelial cancer cells use oxidative mitochondrial metabolism. Cell Cycle 9, 3506-3514.

Bonuccelli, G.,Whitaker-Menezes, D., Castello-Cros, R., Pavlides, S., Pestell, R. G., Fatatis, A., Witkiewicz, A. K., Vander Heiden, M. G., Migneco, G., Chiavarina, B., Frank, P. G., Capozza, F., Flomenberg, N., MartinezOutschoorn, U. E., Sotgia, F., Lisanti, M. P. (2010b) The reverse Warburg effect. Glycolysis inhibitors prevent the tumor promoting effects of caveolin-1 deficient cancer associated fibroblasts. Cell Cycle $\mathbf{9}$, 1960-1971.

Chen, L. B. (1988) Mitochondrial membrane potential in living cells. Annu. Rev. Cell Biol. 4, 155-181.

Chernet, B. T., Levin, M. (2014) Transmembrane voltage potential of somatic cells controls oncogenemediated tumorigenesis at long-range. Oncotarget 5, 3287-3306.

Chiavarina, B., Whitaker-Menezes, D., Migneco, G., Martinez-Outschoorn, U. E., Pavlides, S., Howell, A., Tanowitz, H. B., Casimiro, M. C., Wang, C., Pestell, R. G., Grieshaber, P., Caro, J., Sotgia, F., Lisanti, M. P. (2010) HIF1-alpha functions as a tumor promoter in cancer associated fibroblasts, and as a tumor suppressor in breast cancer cells. Autophagy drives compartment-specific oncogenesis. Cell Cycle 9, 3534-3551.

Fröhlich, H. (1968a) Bose condensation of strongly excited longitudinal electric modes. Phys. Lett. A 26, 402-403.

Fröhlich, H. (1968b) Long-range coherence and energy storage in biological systems. Int. J. Quantum Chem. II, 641-649.

Fröhlich, H. (1969) Quantum mechanical concepts in biology. In: Theoretical Physics and Biology, ed. Marois, M., Pp. 13-22, North Holland, Amsterdam (Proc. $1^{\text {st }}$ Int. Conf. Theor. Phys. Biol., Versailles, 1967).

Fröhlich, H. (1973) Collective behaviour of non-linearly coupled oscillating fields (with applications to biological systems). J. Collect. Phenom. 1, 101-109.

Fröhlich, H. (1978) Coherent electric vibrations in biological systems and cancer problem. IEEE Trans. MTT 26, 613-617.

Fröhlich, H. (1980) The biological effects of microwaves and related questions. Adv. Electronics Electron Phys. 53, 85-152.

Goldstein, S., Korczack, L. B. (1981) Status of mitochondria in living human fibroblasts during growth and senescence in vitro: Use of the laser dye rhodamine 123. J. Cell Biol. 91, 392-398.

Heerdt, B. G., Houston, M. A., Wilson, A. J., Augenlicht, L. H. (2003) The intrinsic mitochondrial membrane potential $\left(\Delta \Psi_{\mathrm{m}}\right)$ is associated with steady-state mitochondrial activity and the extent to which colonic epithelial cells undergo butyrate-mediated growth arrest and apoptosis. Cancer Res. 63, 6311-6319.

Heerdt, B. G., Houston, M. A., Augenlicht, L. H. (2006) Growth properties of colonic tumor cells are a function of the intrinsic mitochondrial membrane potential. Cancer Res. 66, 1591-1596.

Jandová, A., Laurová, L., Novotná, J., Škoda, V. (1969a) The level of the fifth isoenzyme LDH in the blood serum in precancerous endometrium patients. Cesk. Gynekol. 34, 402. (in Czech)

Jandová, A., Škoda, V., Lavrová, L. (1969b) La frequence d'isozyme LDH dans le serum sanguin constatee, chez les femmes souffrant de cancer de la vulve. C. R. Soc. Fr. Gyncol. 39, 418-419.

Pokorný J.; Pokorný J.; Kobilková J.; Jandová A.;Vrba J.;Vrba J. Jr. 
Jandová, A., Miluničová, A., Škoda, V. (1970) Relation of isoenzyme LDH to hematological values in gynecological carcinoma. Transfúze 4, 91-92. (in Czech)

Jandová, A., Kubátová, A., Macků, F., Novotná, J., Pezlarová, A. (1971) LDH activity in blood serum and tumorous tissue in ovarial cancer patients. Cesk. Gynekol. 36, 549-550. (in Czech)

Jandová, A., Hurych, J., Pokorný, J., Čoček, A., Trojan, S., Nedbalová, M., Dohnalová, A. (2001) Effects of sinusoidal magnetic field on adherence inhibition of leukocytes. Electro Magnetobiol. 20, 397-413.

Jandová, A., Pokorný, J., Kobilková, J., Janoušek, M., Mašata, J., Trojan, S., Nedbalová, M., Dohnalová, A., Beková, A., Slavík, V., Čoček, A., Sanitrák, J. (2009) Cell-mediated immunity in cervical cancer evolution. Electromagn. Biol. Med. 28, 1-14.

Johnson, L.V., Summerhayes, I. C., Chen, L. B. (1982) Deceased uptake and retention of rhodamine 123 by mitochondria in feline sarcoma virus-transformed mink cells. Cell 28, 7-14.

Kaplon, J., Zheng, L., Meissl, K., Chaneton, B., Serlivanov, V. A., Mackay, G., van der Burg, S. H., Verdegaal, E. M. E., Cascante, M., Shlomi, T., Gottlieb, E., Peeper, D. S. (2013) A key role for mitochondrial gatekeeper pyruvate dehydrogenase in oncogene-induced senescence. Nature 498, 109-112.

Klingenberg, M., Rottenberg, H. (1977) Relation between the gradient of the ATP/ADP ratio and the membrane potential across the mitochondrial membrane. Eur. J. Biochem. 73, 125-130.

Ko, Y. H., Lin, Z., Flomenberg, N., Pestell, R. G., Howell, A., Sotgia, F., Lisanti, M. P., Martinez-Outschoorn, U. E. (2011) Glutamine fuels a vicious cycle of autophagy in the tumor stroma and oxidative mitochondrial metabolism in epithelial cancer cells. Implications for preventing chemotherapy resistance. Cancer Biol. Ther. 12, 1085-1097.

Lampidis, T. J., Bernal, S. D., Summerhayes, I. C., Chen, L. B. (1983) Selective toxicity of rhodamine 123 in carcinoma cells in vitro. Cancer Res. 43, 716-720.

Levin, M. (2012) Morphogenetic fields in embryogenesis, regeneration, and cancer: Non-local control of complex patterning. Biosystems 109, 243-261.

Levin, M. (2014) Endogenous bioelectrical networks store non-genetic patterning information during development and regeneration. J. Physiol. 592, 2295-2305.

Lisanti, M. P., Martinez-Outschoorn, U. E., Chiavarina, B., Pavlides, S., Whitaker-Menezes, D., Tsirigos, A., Witkiewicz, A., Lin, Z., Balliet, R., Howell, A., Sotgia, F. (2010) Understanding the "lethal" drivers of tumor-stroma co-evolution. Emerging role(s) for hypoxia, oxidative stress and autophagy/mitophagy in the tumor micro-environment. Cancer Biol. Ther. 10, 537-542.

Martinez-Outschoorn, U. E., Balliet, R. M., Rivadeneira, D. B., Chiavarina, B., Pavlides, S., Wang, C., WhitakerMenezes, D., Daumer, K. M., Lin, Z., Witkiewicz, A. K., Flomenberg, N., Howell, A., Pestell, R. G., Knudsen, E. S., Sotgia, F., Lisanti, M. P. (2010a) Oxidative stress in cancer associated fibroblasts drives tumor-stroma co-evolution. A new paradigm for understanding tumor metabolism, the field effect and genomic instability in cancer cells. Cell Cycle 9, 3256-3276.

Martinez-Outschoorn, U. E., Trimmer, C., Lin, Z., Whitaker-Menezes, D., Chiavarina, B., Zhou, J., Wang, C., Pavlides, S., Martinez-Cantarin, M. P., Cappozza, F., Witkiewicz, A. K., Flomenberg, N., Howell, A., Pestell, R. G., Caro, J., Lisanti, M. P., Sotgia, F. (2010b) Autophagy in cancer associated fibroblasts promotes tumor cell survival. Role of hypoxia, HIF1 induction and NFKB activation in the tumor stromal microenvironment. Cell Cycle 9, 3515-3533.

Martinez-Outschoorn, U. E., Lin, Z., Ko,Y. H., Goldberg, A. F., Flomenberg, N., Wang, C., Pavlides, S., Pestell, R. G., Howell, A., Sotgia, F., Lisanti, M. P. (2011) Understanding the metabolic basis of drug resistance. Therapeutic induction of the Warburg effect kills cancer cells. Cell Cycle 10, 2521-2528.

Migneco, G., Whitaker-Menezes, D., Chiavarina, B., Castello-Cros, R. C., Pavlides, S., Pestell, R. G., Fatatis, A., Flomenberg, N., Tsirigos, A., Howell, A., Martinez-Outschoorn, U. E., Sotgia, F., Lisanti, M. P. (2010) 
Glycolytic cancer associated fibroblasts promote breast cancer tumor growth, without a measurable increase in angiogenesis. Evidence for stromal-epithelial metabolic coupling. Cell Cycle 9,

2412-2422.

Modica-Napolitano, J. S., Aprille, J. R. (1987) Basis for selective cytotoxicity of rhodamine 123. Cancer Res. 47, 4361-4365.

Modica-Napolitano, J. S., Weiss, M. J., Chen, L. B., Aprille, J. R. (1984) Rhodamine 123 inhibits bioenergetic function in isolated rat liver mitochondria. Biochem. Biophys. Res. Commun. 118, 717-723.

Modica-Napolitano, J. S., Steele, G. D. Jr., Chen, L. B. (1989) Aberrant mitochondria in two human colon carcinoma cell lines. Cancer Res. 49, 3369-3373.

Modica-Napolitano, J. S., Koya, K., Weisberg, E., Brunelli, B. T., Li, Y., Chen, L. B. (1996) Selective damage to carcinoma mitochondria by the rhodacyanine MKT-077. Cancer Res. 56, 544-550.

Nadakavukaren, K. K., Nadakavukaren, J. J., Chen, L. B. (1985) Increased rhodamine 123 uptake by carcinoma cells. Cancer Res. 45, 6093-6099.

O’Connor, J. E., Vargas, J. L., Kimler, B. F., Hernandez-Yago, J., Grisolia, S. (1988) Use of rhodamine 123 to investigate alterations in mitochondrial activity in isolated mouse liver mitochondria. Biochem. Biophys. Res. Commun. 151, 568-573.

Olenchock, B. A., Vander Heiden, M. G. (2013) Pyruvate as a pivot point for oncogene-induced senescence. Cell 153, 1429-1430.

Pavlides, S., Whitaker-Menezes, D., Castello-Cros, R., Flomenberg, N., Witkiewicz, A. K., Frank, P. G., Casimiro, M. C., Wang, C., Fortina, P., Addya, S., Pestell, R. G., Martinez-Outschoorn, U. E., Sotgia, F., Lisanti, M. P. (2009) Reverse Warburg effect. Aerobic glycolysis and cancer associated fibroblasts and their tumor stroma. Cell Cycle 8, 3984-4001.

Pavlides, S., Tsirigos, A., Migneco, G., Whitaker-Menezes, D., Chiavarina, B., Flomenberg, N., Frank, P. G., Casimiro, M. C., Wang, C., Pestell, R. G., Martinez-Outschoorn, U. E., Howell, A., Sotgia, F., Lisanti, M. P. (2010a) The autophagic tumor stroma model of cancer. Role of oxidative stress and ketone production in fuelling tumor cell metabolism. Cell Cycle 9, 3485-3505.

Pavlides, S., Tsirigos, A., Vera, I., Flomenberg, N., Frank, P. G., Casimiro, M. C., Wang, C., Fortina, P., Addya, S., Pestell, R. G., Martinez-Outschoorn, U. E., Sotgia, F., Lisanti, M. P. (2010b) Loss of stromal caveolin-1 leads to oxidative stress, mimics hypoxia and drives inflammation in the tumor microenvironment, conferring the "reverse Warburg effect". A transcriptional informatics analysis with validation. Cell Cycle 9, 2201-2219.

Pohl, H. A., Braden, T., Robinson, S., Piclardi, J., Pohl, D. G. (1981) Life cycle alterations of the microdielectrophoretic effects of cells. J. Biol. Phys. 9, 133-154.

Pokorný, J. (2012) Physical aspects of biological activity and cancer. AIP Adv. 2, 011207-1-11.

Pokorný, J., Hašek, J., Jelínek, F., Šaroch, J., Palán, B. (2001) Electromagnetic activity of yeast cells in the M phase. Electro Magnetobiol. 20, 371-396.

Pokorný, J., Vedruccio, C., Cifra, M., Kučera, O. (2011) Cancer physics: diagnostics based on damped cellular elastoelectrical vibrations in microtubules. Eur. Biophys. J. 40, 747-759.

Pokorný, J., Cifra, M., Jandová, A., Kučera, O., Šrobár, F., Vrba, J., Vrba, J. Jr., Kobilková, J. (2012a) Targeting mitochondria for cancer treatment. Eur. J. Oncol. 17, 23-36.

Pokorný, J., Jandová, A., Nedbalová, M., Jelínek, F., Cifra, M., Kučera, O., Havelka, D., Vrba, J., Vrba, J. Jr., Čoček, A., Kobilková, J. (2012b) Mitochondrial metabolism - Neglected link of cancer transformation and treatment. Prague Med. Rep. 113, 81-94.

Pokorný, J., Foletti, A., Kobilková, J., Jandová, A., Vrba, J., Vrba, J. Jr., Nedbalová, M., Čoček, A., Danani, A., Tuszyński, J.A. (2013a) Biophysical insights into cancer transformation and treatment. Scientific World Journal 2013, doi:10.1155/2013/195028.

Pokorný J.; Pokorný J.; Kobilková J.; Jandová A.;Vrba J.;Vrba J. Jr. 
Pokorný, J., Pokorný, J., Kobilková, J. (2013b) Postulates on electromagnetic activity in biological systems and cancer. Integr. Biol. (Camb.) 5, 1439-1446.

Pokorný, J., Pokorný, J., Kobilková, J., Jandová, A., Vrba, J., Vrba, J. Jr. (2014) Cancer - Pathological breakdown of coherent energy states. Biophys. Rev. Lett. 9, doi:10.1142/S1793048013300077.

Reed, P.W. (1979) lonophores. Methods Enzymol. 55, 435-454.

Sahu, S., Ghosh, S., Ghosh, B., Aswani, K., Hirata, K., Fujita, D., Bandyopadhyay, A. (2013) Atomic water channel controlling remarkable properties of a single brain microtubule: Correlating single protein to its supramolecular assembly. Biosens. Bioelectron. 47, 141-148.

Seemayer, T. A., Lagacé, R., Schürch, W., Tremblay, G. (1979) Myofibroblasts in the stroma of invasive and metastatic carcinoma. Am. J. Surg. Pathol. 3, 525-533.

Škoda, V., Trnková, M., Škramovský, V., Jandová, A., Novotná, J. (1967) Changes of the isoenzyme of lactate dehydrogenase acid (LDH 5) in blood serum in course of cytostatic treatment of gynecological cancer. Cesk. Gynekol. 32, 14-17. (in Czech)

Sotgia, F., Whitaker-Menezes, D., Martinez-Outschoorn, U. E., Flomenberg, N., Birbe, R. C., Witkiewicz, A. K., Howell, A., Philp, N. J., Pestell, R. G., Lisanti, M. P. (2012) Mitochondrial metabolism in cancer metastasis. Visualizing tumor cell mitochondria and the "reverse Warburg effect" in positive lymph node tissue. Cell Cycle 11, 1445-1454.

Soto, A. M., Sonnenschein, C. (2011) The tissue organization field theory of cancer: A testable replacement for the somatic mutation theory. Bioessays 33, 332-340.

Sun, R. C., Fadia, M., Dahlstrom, J. E., Parish, C. R., Board, P. G., Blackburn, A. C. (2010) Reversal of the glycolytic phenotype by dichloroacetate inhibits metastatic breast cancer cell growth in vitro and in vivo. Breast Cancer Res. Treat. 120, 253-260.

Tyner, K. M., Kopelman, R., Philbert, M. A. (2007) “Nanosized voltmeter” enables cellular-wide electric field mapping. Biophys. J. 93, 1163-1174.

Vedruccio, C., Meessen, A. (2004) EM cancer detection by means of non-linear resonance interaction. In: Proceedings PIERS, Progress in Electromagnetic Research Symposium, Pisa, Italy, March 28-31, 2004, pp. 909-912.

Warburg, O. (1956) On the origin of cancer cells. Science 123, 309-314.

Warburg, O., Posener, K., Negelein, E. (1924) Über den Stoffwechsel der Carcinomzelle. Biochem. Z. 152, 309-344.

Witkiewicz, A. K., Whitaker-Menezes, D., Dasgupta, A., Philp, N. J., Lin, Z., Gandara, R., Sneddon, S., MartinezOutschoorn, U. E., Sotgia, F., Lisanti, M. P. (2012) Using the "reverse Warburg effect" to identify high-risk breast cancer patients. Stromal MCT4 predicts poor clinical outcome in triple-negative breast cancers. Cell Cycle 11, 1108-1117.

Zarbl, H., Latreille, J., Jolicoeur, P. (1987) Revertants of v-fos-transformed fibroblasts have mutations ion cellular genes essential for transformation by other oncogenes. Cell 51, 357-369.

Zheng, J. M., Pollack, G. H. (2003) Long-range forces extending from polymer-gel surfaces. Phys. Rev. E Stat. Nonlin. Soft Matter Phys. 68, 031408.

Zheng, J. M., Pollack, G. H. (2006) Solute exclusion and potential distribution near hydrophilic surfaces. In: Water and the Cell, eds. Pollack, G. H., Cameron, I., Wheatley, D. N., Pp. 165-174, Springer, Dordrecht. 